Mediterránea, 5 - Págs. (23 - 33), Año 1981

\title{
AVES ACUÁTICAS \\ DE LA MAR CHICA DE MELILLA, JULIO 1977 - JUNIO 1978 Y VERANO 1979
}

\author{
Por J. M. Cabo' e I. Camacho'
}

\section{INTRODUCCION}

La laguna costera llamada Mar Chica, está situada a unos 3 $\mathrm{Kms}$. de la ciudad de Melilla, orientada en dirección E-SE; tiene forma eliptica, con unos $25 \mathrm{Kms}$. en su eje mayor y 6:7 Kms. en su eje menor. (Fig. 1)

El borde oriental está formado por una barrera de arena de unos 300-400 m. de ancho, llamada Restinga, interrumpida en un punto que lo pone en comunicación con el Mar Mediterráneo, llamada Bocana. Su profundidad máxima se alcanza frente a Nador, con unos $7 \mathrm{~m}$.

La red hidrográfica es muy escasa, con unos pocos rios y arroyos de curso estacional y carácter torrencial, que sólo en ocasiones llegan a verter sus aguas en ella.

\section{MATERIAL Y METODOS}

Se ha hecho una serie de observaciones desde el mes de julio de 1977 hasta junio de 1978, excepto los meses de septiembre, abril y mayo, que aparecen en el cuadro resumen con un guión en su casiIla correspondiente, y posteriormente en julio, agosto y septiembre de 1979. Estos últimos meses nos han servido para confirmar algunos datos que consideramos dudosos y que habiamos observado anteriormente.

(1) Departamento de Zoología. Universidad de Granada. 


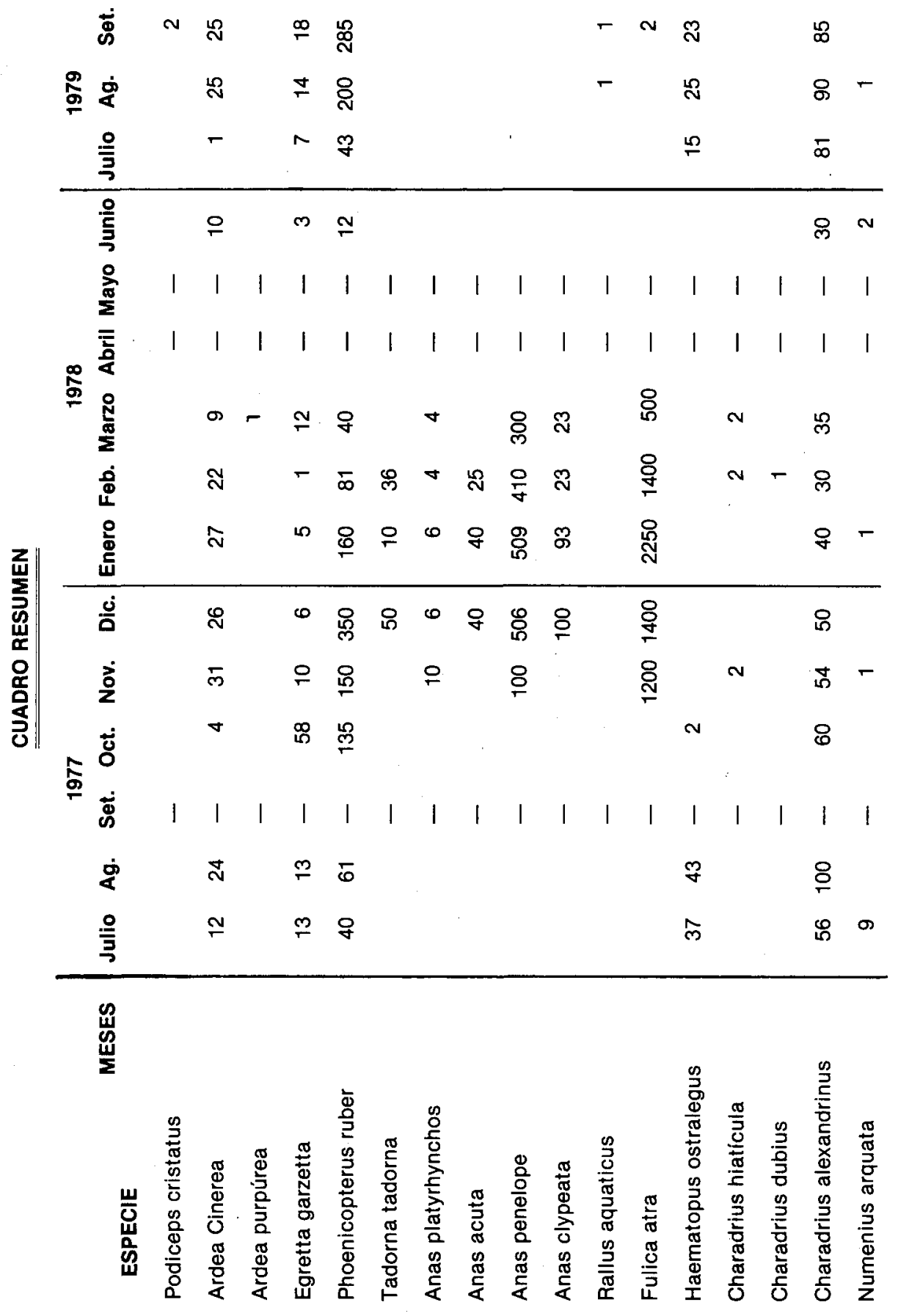




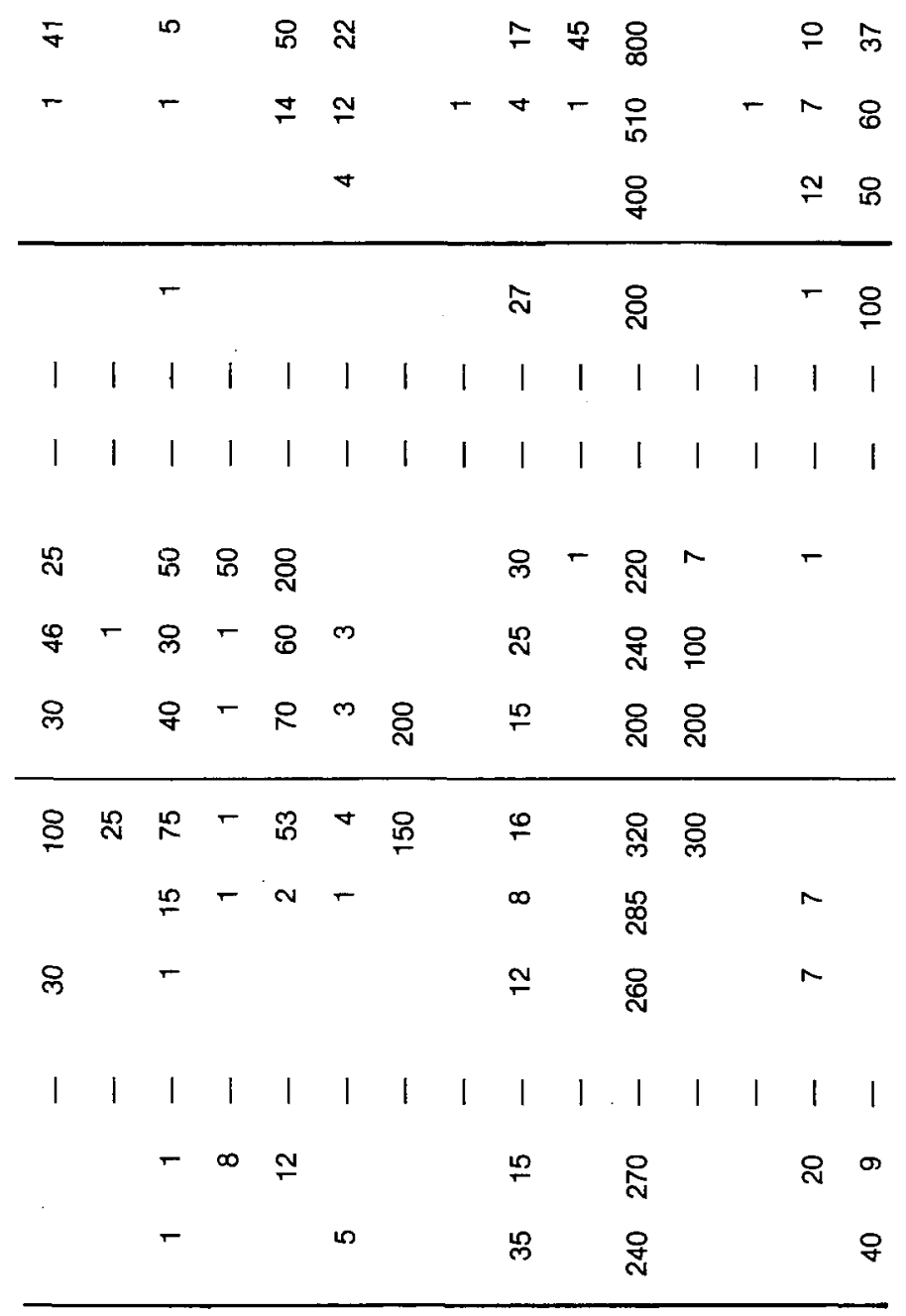

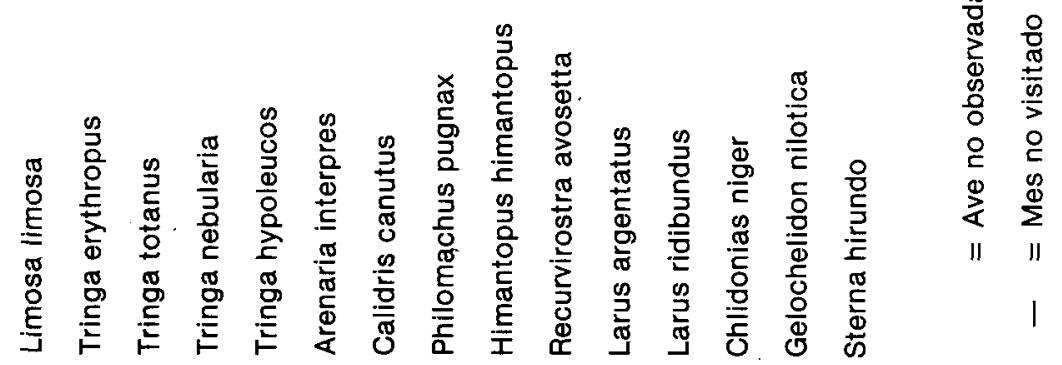




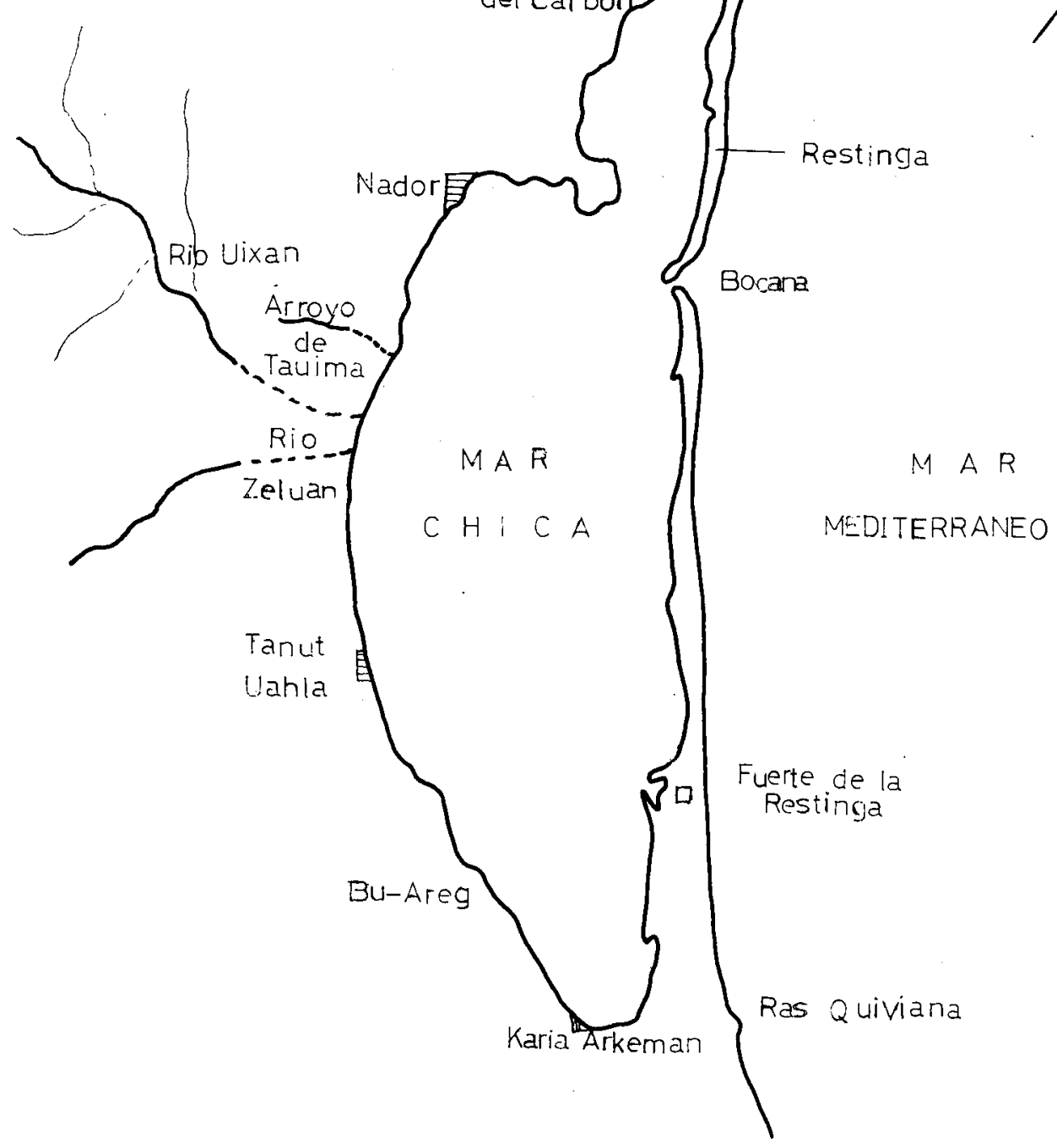

Fig. I 
Las visitas no se han hecho de modo sistemático y regular, no obstante se pueden calcular en una media de 3 veces por mes.

Las zonas más interesantes desde el punto de vista ornitológico resultaron ser (Fig. I):

1) Karia-Arkeman: En el extremo inferior S-SE donde se encuentran unas salinas hoy abandonadas, con zonas encharcadas y manchas de vegetación halófila. Estas salinas están limitadas por el N-NE por bosques de eucaliptos y por el S-SO por campos de cultivos.

2) Muelle del Carbón: Situada en el extremo opuesto al anterior. Formada de arenales-fangosos con manchas ralas de vegetación halófila.

3) La Bocana: Situada en la Restinga y que en este último verano, como consecuencia de la formación de bancos de arena, ha adquirido mayor interés que en los años anteriores al ser visitado éste por gran número de especies acuáticas. Presenta una vegetación halófila en su margen interior (orilla de La Mar Chica) y de dunas en su margen externo (orilla del Mediterráneo).

Las visitas a las tres zonas descritas se hacian el mismo día, excepto en alguna ocasión en que sólo se ha visitado alguna de ellas. El recorrido en cada una se hacía a pie y utilizando prismáticos.

\section{RESULTADOS}

En el cuadro resumen se recoge el número máximo de aves observado en el conjunto de visitas llevadas a cabo cada uno de los meses.

La gráfica I muestra la evolución de la máxima población observada a lo largo de dicho periodo. Destacan en la misma las cifras invernales próximas a los 4.000 individuos, mientras que en verano apenas llegan a 500 en julio de 1977.

\section{DISCUSION SOBRE ALGUNA DE LAS ESPECIES}

En las visitas realizadas en verano hemos observado la presencia de algunas aves fundamentalmente migratorias, algunas de ellas susceptibles de confusión con algunas otras, por su gran parecido, como ocurre con algunos limícolos como Tringa nebularia y $T$. totanus, otras en cambio no, como agujas y vuelvepiedras por su característica coloración estival. No obstante, el verano de 1979 se han hecho observaciones para cerciorarse de la presencia de algunos de ellos. La confirmación llevada a cabo va avalada por datos 


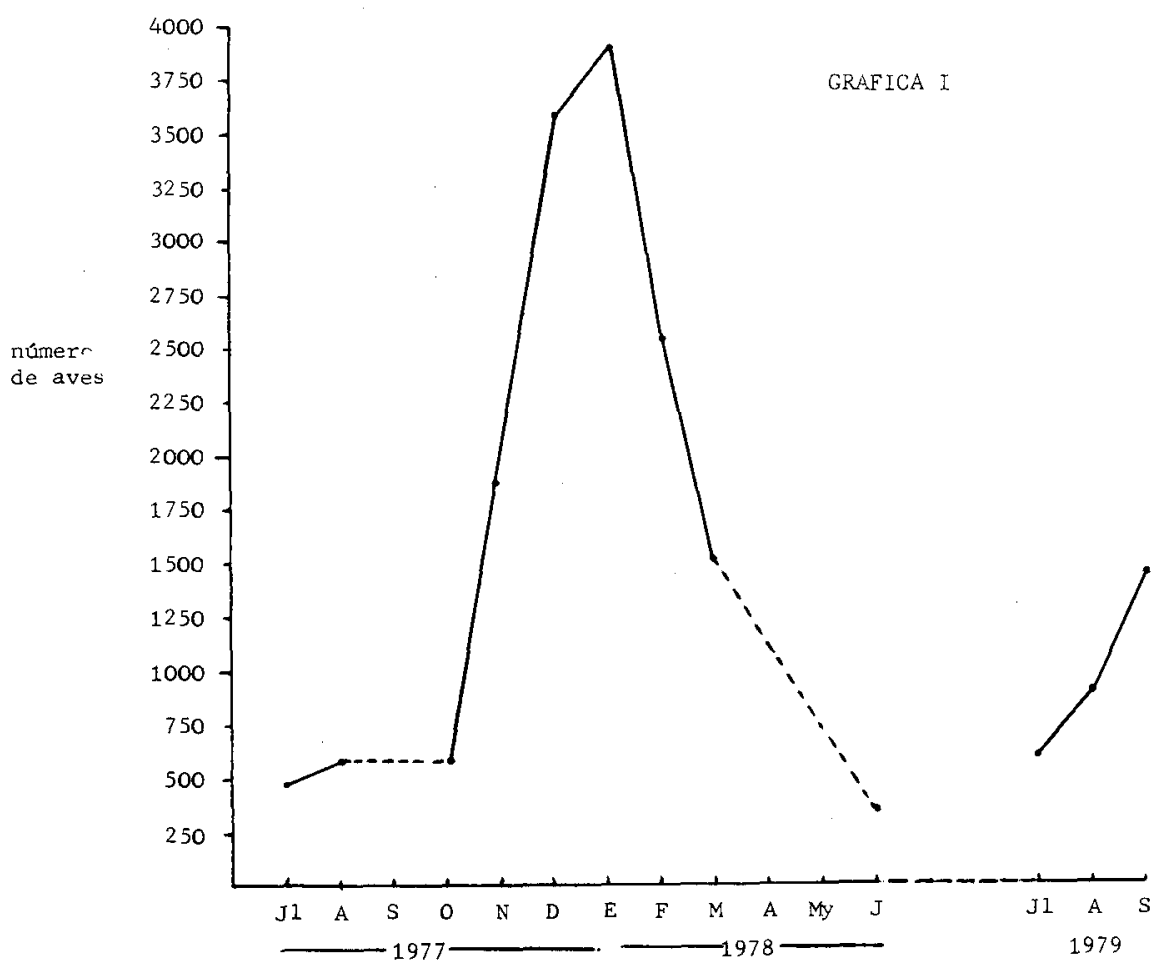

que hemos encontrado en la bibliografia y que a continuación se citan:

\section{Fulica atra L. (Focha común)}

Llama la atención su ausencia, al menos en las observaciones llevadas a cabo en verano, no obstante conocerse varios lugares de nidificación en el N-W de Africa, Bernis (1966). Esta ausencia, real o aparente, quizá sea debida al carácter más receloso en la época de cría, que la haría menos visible al buscar protección entre la vegetación.

Las cifras invernales, por el contrario, la muestran como la más abundante entre todas las aves acuáticas, aspecto semejante al que ocurre en la avifauna acuática europea.

Haematopus ostralegus L. (Ostrero)

En numerosas ocasiones hemos podido observar la presencia estival del ostrero. Algunas de ellas son: en el año 1977, el 25-VII, 15 individuos; el 27-VII, 9 y el 29-VII, 37; el día 10-VIII, 43; el 16-VIII, 40 y el 19-VIII, 37. 
En el año 1979, el 23-VII, 15 individuos; el 3-VIII, 25; el 23-VIII, 22 $y$ el 4-IX, 23.

La presencia de esta ave ha estado ligada casi indefectiblemente al Muelle del Carbón y Restinga, formado por fondos fangosos y arenosos. Sólo hay una cita en Karia-Arkeman, en septiembre, con 1 solo individuo de los 23 observados.

VALVERDE (1956), cita en julio uno en la desembocadura del Lucus (Larache) y también se le ha citado nidificando en Cabo $\mathrm{Ne}$ gro.

Numenius arquatus L. (Zarapito real)

Poco abundante y repartido a lo largo de todo el año. Las citas estivales encontradas han sido 25-VII-1977, 9; el 20-VI-1978, 2 y el 11 y 23 del VIII de 1979, 1 en cada ocasión.

Según ETCHECOPAR y HÜE (1964) se encuentra presente en Marruecos a lo largo de todo el año, ya que probablemente los inmaduros permanecen muy al sur de su área de nidificación. En el sur de España también se han observado intentos de reproducción, BERNIS (1966).

\section{Limosa limosa L. (Aguja colinegra)}

Sólo se ha observado en agosto y septiembre de 1979. De las tres visitas de agosto, los días 3,11 y 23, sólo se vío un individuo el dia 11, mientras que el 4-IX-79, 41.

ETCHECOPAR y HÜE (1964) y BERNIS (1966) manifiestan que quedan individuos no nidificantes en diferentes latitudes. Por otro lado VALVERDE (1956) cita unos 2.000 individuos en migración activa postnupcial en el Guadalquivir el 24 de junio.

Tanto Tringa totanus como T.hypoleucos su presencia estival no es tan rara ya que aunque migradores se conocen citas de nidificación en el sur de la Península, así como también en Marruecos, BERNIS (1966) y ETCHECOPAR (1964).

Arenaria interpres L. (Vuelvepiedras)

Tenemos anotaciones estivales de julio, agosto y septiembre. Por otra parte su reconocimiento no ofrece duda debido a su típica coloración estival.

Citas españolas de agosto de 1969 se pueden ver en VAUGHAN (1971) en Santa Pola (Alicante), con 2 individuos. Otras más meridionales, también de agosto, son recuperaciones en Cádiz y Lanzarote, BERNIS (1966). 


\section{ALGUNOS DATOS SOBRE NIDIFICACION}

Aunque el trabajo ha sido orientado más hacia la variedad de la avifauna y en especial la presencia estival de algunas de ellas, se han recogido sólo unos pocos datos de reproducción de algunas de ellas ya que los meses de abril y mayo, que son clave para los estudios de esta índole no se han podido visitar.

Las especies cuya nidificación se ha comprobado han sido 3 :

De Phoenicopterus ruber (L.) se observaron 5 pollos, que aún no volaban bien, el 4-IX-1979. Además tenemos datos del día 25-VII1977 con 3 inmaduros y también el 19-VIII se vieron inmaduros aunque no se anotó su número, pero era bajo.

Aunque no se ha comprobado materialmente la nidificación, la presencia de inmaduros en fechas tempranas como en julio nos lleva a asegurar que nidifican aunque en pequeña cantidad ya que de otro modo no se podría explicar la presencia aquí de estas aves. Por otra parte la observación de jóvenes que aún no volaban bien apoyan esta aseveración.

Charadrius alexandrinus L. (Chorlitejo patinegro)

El 20-VI-1978 se vieron varias parejas con actitudes reproductoras en la Bocana y observándose 3 pollos que aún no volaban.

\section{Himantopus himantopus L. (Cigüeñuela)}

El 4-IX-1979 se vió un pollo volantón en Karia-Arkeman. En años anteriores ya se habia visto una conducta reproductora que hizo sospechar su nidificación.

\section{Sterna hirundo L. (Charrán común)}

A finales de junio de 1978 se capturó un pollo que seguidamente se puso en libertad.

\section{RESUMEN Y CONCLUSIONES}

En nuestras observaciones se ve que a partir de julio, aumenta la población, debido al incremento sufrido como consecuencia de la reproducción y que se verá aumentada con la llegada de los invernantes especialmente a partir de octubre.

En enero comienza un descenso brusco, debido a la disminución de Fulica atra y que prosigue con la partida de los nidificantes septentrionales, hasta llegar a junio que registra las cifras más bajas del año. Algunos de estos típicos nidificantes dejan algunos individuos en esta zona, destacando entre ellos: Haematopus ostralegus, Numenius arquatus, Limosa limosa, Tringa totánus, T. hypoleucos y Arenaria interpres. 
En total se han encontrado 32 especies diferentes de aves acuáticas, observándose la máxima variedad desde diciembre a marzo. La mayor abundancia ha correspondido a los mismos meses con cifras superiores a los 4.000 individuos en enero e inferiores a los 500 en julio de 1977.

Los Laro-limicolos son los que aportan mayor variedad con un total de 20 especies diferentes, de las que 15 corresponden a Limícolas y 5 a Láridos. De los primeros, destacan por su número: Charadrius alexandrinus, Limosa limosa, Tringa totanus, T. hypoleucós, Calidris canutus e Himantopus himantopus; de los segundos: Larus argentatus y $L$. ridibundus.

Finalmente las especies más abundantes, en orden numérico decreciente fueron: Fulica atra, Anas penélope, Larus argentatus, $L$. ridibundus y Phoenicopterus ruber. 


\section{RESUMEN}

Durante 1978 y 1979 se hizo una serie de observaciones sobre aves acuáticas en la Mar Chica (Melilla).

En conjunto se contaron 32 especies diferentes, siendo la más abundante: Charadrius alexandrinus, Limosa limosa, Tringa totanus y $T$. hypoleucos. Algunas de ellas, así como Haematopus ostralegus y Arenaria interpres dejan algunos individuos en época invernal.

La especie más abundante fue Fulica atra, con más de 2.000 individuos en enero.

\section{SUMMARY}

Some observations on waterfowl in the Mar Chica (Melilla) during 1978 and 1979, was made.

32 different species has been seen, being the four more abundant: Charadrius alexandrinus, Limosa limosa, Tringa totanus and $T$. hypoleucos. A few of them, as well as some Haematopus ostralegus and Arenaria interpres remain in winter season.

The most abundant species was Fulica atra, with more than 2.000 specimens in january. 


\section{BIBLIOGRAFIA}

BERNIS, F. (1966): Aves migradoras ibéricas. Fascículo $4 .^{\circ}, 503-705$. Ed. S.E.O. Madrid.

ETCHECOPAR, R.D. \& HÜE, F., (1964): Les Oiseaux du Nord de L'Afrique. Ed. N. Boubée y Cie. 606 pag. Paris.

JUANA, E. de, (1976): Nuevos datos de invierno sobre aves de Marruecos. Ardeo/a, Vol. 23: 49-62.

VALVERDE, J.A., (1955): Aves de Marruecos español en Julio. Ardeola, Vol. II, n. ${ }^{\circ} 1: 87-114$.

(1956): Aves de Marruecos español en Julio. Ardeola, Vol. II, n. ${ }^{\circ}$ 2: 213-240.

VÁUGHAN, R., (1971): Nota sobre aves acuáticas observadas en las salinas de Santa Pola (Alicante), 20-30 de agosto 1969. Ardeola, Vol. 15: 94. 